\title{
On conformal transformations of Riemannian spaces.
}

\author{
By Tadashi NAGANO
}

(Received Sept. 20, 1957)

Introduction. In this paper, we mean by a space always a connected Riemannian space (with a positive definite metric) of class $C^{\infty}$ and of dimension $n>3$. We denote such a space generally with $M$, and the conformal transformation group of $M$ with $C(M)$, the isometry group of $M$ with $I(M)$. If $M^{\prime}$ is another space and $C(M) \subset I\left(M^{\prime}\right)$, the study of $C(M)$ will be reduced to that of $I\left(M^{\prime}\right)$. We shall say for brevity that $M$ is conformally reducible if we can find an $M^{\prime}$ with $C(M) \subset I\left(M^{\prime}\right)$. Several authors have shown that spaces satisfying some conditions on the Ricci tensor have the property $C(M) \subset I(M)$ or $C^{0}(M) \subset I(M)$, where $C^{0}(M)$ means the identity component of $C(M)$ (see [8]). In $\S 1$ of this paper, we shall show that $C(M) \subset I\left(M^{\prime}\right)$ holds for a submanifold $M^{\prime}$ of $M$ whenever $M$ is not conformally flat. Thus, spaces which are not conformally flat, are conformally reducible in the sense explained above. This is based on a theorem (theorem $\mathrm{H}$ ) essentially due to Hlavatý. From this remark follow very easily the following known results for example:

I. If an $n$-dimensional Riemannian space $M(n>4)$ admits the group $C(M)$ of conformal transformations with dimensions greater than $n(n-1) / 2$ +7 , then $M$ is conformally flat (H. Hiramatu [1]).

II. Let $\rho$ be the natural representation on the tangent space $T_{p}$ of $M$ at a point $p \in M$ of the group $C_{p}(M)$ of isotropy of $C(M)$ at $p$. If $\rho\left(C_{p}(M)\right)$ is not contained in the rotation group of $T_{p}$ with the induced metric, the conformal tensor vanishes at $p$ (S. Ishihara and M. Obata, see [8] p. 277).

As another application of theorem $\mathrm{H}$ we shall prove that a Riemannian homogeneous space which is not conformally flat does not admit a conformal transformation but for an isometry.

In $\$ \S 2-4$, we shall consider conformally flat spaces, to which the "method of reduction" is not applicable. If we denote the $n$-Möbius group (for definition see below) by $M(n)$, the contents of $\S 2$ may be summarized by the following three statements:

(1) We have always $C(M) \subset M(n)$ locally,

(2) $C(M)=M(n)$ locally if and only if $M$ is conformally equivalent with $S^{n}$ ( $S^{n}$ means the $n$-sphere, cf. [4]).

(3) If $C(M)$ is transitive, then there exists an open submanifold $M^{\prime}$ 
of $S^{n}$, such that the universal covering space $\tilde{M}$ of $M$ is conformally equivalent with $\tilde{M}^{\prime}$.

In $\S \S 3-4$, we determine the structure of $M$ such that $\operatorname{dim} M>n(n-1) / 2$ +2 . $\S 3$ treats the case where $C(M)$ is transitive on $M$, and $\S 4$ the intransitive case. Our main results in this respect are theorems 9 and 10 .

In this paper, we use the notations and the results of Yano [8] without special references. The author wishes to express his sincere thanks to Prof. K. Yano and Mr. M. Obata, for their constant encouragements and valuable suggestions during the preparation of this paper.

\section{$\S 1$. Reduction to the isometry group.}

A diffeomorphism $\tau$ of a Riemannian space $M$ into another Riemannian space $M^{*}$ is, by definition, conformal, if $\tau$ preserves the orthogonality, or equivalently, if there exists a positive scalar $f$ on $M$ such that the length $\|u\|$ of a tangent vector $u$ at $x \in M$ and the length $\|d \tau(u)\|$ of $d \tau(u)$ enjoy the relation

$$
\|d \tau(u)\|^{*}=f(x)\|u\| \text {. }
$$

Moreover if $\tau$ maps $M$ onto $M^{*}, M$ is said conformally equivalent to $M^{*}$. Further if $M$ coincides with $M^{*}, \tau$ is called a conformal transformation of $M$. All the conformal transformations of $M$ form a Lie transformation group of $M$. There exists on $M$ a $(1,3)$-type tensor field $C$ known as Welyl's conformal curvature tensor (in short, the conformal tensor), which is invariant under any conformal transformation of $M$. An infinitesimal conformal transformation $u$ is a vector field on $M$ such that $£_{u} g=2 \phi g$, i. e. the Lie derivative of the fundamental tensor $g$ with respect to $u$ is a scalar $2 \phi$ times $g$. The vector $u$ is a Killing vector if in particular $\phi=0$. When $u$ generates a one-parameter group $G$ of transformation of $M$, in order that $G$ consists of conformal transformations it is necessary and sufficient that $u$ is an infinitesimal conformal transformation. The conformal tensor $C$ is invariant by any infinitesimal conformal transformation $u$, i. e. $£_{u} C=0$. Now we have a theorem essentially due to V. Hlavatý [2], though he did not allude to conformal transformations.

TheOREM H. If the conformal tensor $C$ does not vanish at any point of a space $M, M$ is conformally equivalent to a space $M^{*}$ such that the group of conformal transformations on $M$ is naturally isomorphic onto the group of isometries on $M^{*}$. Further $M^{*}$ does not admit an infinitesimal conformal transformation but for a Killing vector field.

Proof. We define a new metric in $M$ by $\|u\|^{*}=\|C\|\|u\|$, where $\|C\|$ is the length of $C$, i.e. 


$$
\|C\|^{2}=C^{a}{ }_{b c d} C^{p}{ }_{q r s} g_{a p} g^{b q} g^{c r} g^{d s} .
$$

Thus we have another space $M^{*}$ which is identical with $M$ as a manifold and is conformally equivalent to $M$. We can readily verify that an arbitrary conformal transformation on $M$ is an isometry of $M^{*}$, the converse being also true. And the analogous holds for an infinitesimal transformation.

We shall show two applications of this theorem $\mathrm{H}$.

Notations. $C(M)$ : the (effective) group of all the conformal transformations of $M$.

$C^{L}(M)$ : the Lie algebra of $C(M)$.

$C^{I}(M)$ : the Lie algebra consisting of the infinitesimal conformal transformations of $M$ and with the usual bracket product.

$I^{I}(M)$ : the Lie algebra of the Killing vectors of $M$.

$C_{p}(M)$ : the subgroup of isotropy of $C(M)$ at a point $p \in M$.

$C_{p}^{I}(M)$ : the subalgebra of $C^{I}(M)$ whose vector fields vanish at $p$.

$C_{p}^{L}(M)$ : the Lie algebra of $C_{p}(M)$.

Then both $C_{p}^{I}(M)$ and $C^{L}(M)$ are subalgebras of $C^{I}(M)$ and we have $C_{p}^{L}(M)=C_{p}^{I}(M) \cap C^{L}(M) . \quad M$ is said conformally flat, if the conformal tensor $C$ vanishes everywhere in $M$.

TheOREM 1. If $M$ is not conformally flat, the conformal group $C(M)$ is isomorphic into the isometry group $I\left(M^{*}\right)$ of some space $M^{*}$.

Proof. Denote by $M_{1}$ the open submanifold of $M$ consisting of the points of $M$ at which we have $C \neq 0$. We shall prove that the conformal group $C(M)$ of $M$ is isomorphic into $C\left(M_{1}\right)$ of $M_{1}$. $\quad M_{1}$ is invariant by $C(M)$ and $C^{I}(M)$. A homomorphism of $C(M)$ into $C\left(M_{1}\right)$ is given by the restriction to $M_{1}$ of each conformal transformation on $M$. The isomorphism (of abstract groups) is proved with the use of conformal circles which are invariant by $C(M)$, if we note that, if three near points of a conformal circle are fixed by a conformal transformation, this leaves the conformal circle invariant point-wise. A homomorphism of $C^{I}(M)$ into $C^{I}\left(M_{1}\right)$ is also given by restriction. It turns out to be an isomorphism because of the uniqueness of the solutions of differential equations satisfied by an arbitrary infinitesimal transformation $u$ (see (2.2) and (2.4) in $\S 2$ ). This isomorphism induces that of $C^{L}(M)$ into $C^{L}\left(M_{1}\right)$, hence the local isomorphism of $C(M)$ into $C\left(M_{1}\right)$, which coincides with the above. Now the theorem follows from theorem $\mathrm{H}$.

Q. E. D.

Theorem 1 means that the conformal transformation group of any space which is not conformally flat can be regarded as a subgroup of the isometry group of a space of the same dimension. Thus it is evident, say, that $M$ is conformally flat if $\operatorname{dim} C(M)>n(n-1) / 2$ by the main theorem in Yano [9] (In his theorem only conformally flat spaces appear. For the case 
where $n=8$, see M. Obata [7]]. A Lie algebra $G^{L}$ consisting of vector fields on $M$ and with the bracket product is said transitive, if the natural map $(u, x) \in G \times M \rightarrow u(x) \in T(M)$ (the tangent bundle of $M$ ) has the image $T(M)$. $M$ is homogeneous (i.e. with a transitive isometry group), if and only if $M$ is complete and $I^{I}(M)$ is transitive.

TheоRем 2. If a space $M$ is not conformally flat and the Killing algebra $I^{I}(M)$ is transitive, then $M$ does not admit a conformal transformation (other than an isometry) nor an infinitesimal one.

Proof. $\|C\|$ is constant, because we have $£_{u}\|C\|=0$ for each $u \in I^{I}(M)$ of connected $M$ and $I^{I}(M)$ is transitive. $M$ being conformally non-flat, the constant $\|C\|$ is not zero. Thus we can apply theorem $H$. Since $\|C\|$ is constant, $M$ and $M^{*}$ in theorem $\mathrm{H}$ have the same isometry group and the same Killing algebra, which must coincide with conformal ones of $M^{*}$ by virtue of theorem $\mathrm{H}$.

Corollary. On a homogeneous Riemannian space $M$ which is not conformally flat, a conformal transformation is an isometry.

This holds more generally for any connected open submanifold of $M$ mentioned above.

Corollary. A locally symmetric space which admits an infinitesimal conformal transformation which is not Killing is a space of constant curvature or the local product of two spaces of constant curvature. And an analogous holds for a symmetric space $M$ and a non-isometric conformal transformation of $M$.

These corollaries were announced in [10].

Theorem 3. Let $M$ be a compact simply connected Riemannian space. If a connected Lie group $G \subset C(M)$ of conformal transformations is transitive, then $M$ is conformally equivalent to a homogeneous space $M^{*}$.

This theorem is an immediate consequence of theorem $\mathrm{H}$ in the case where $M$ is not conformally flat.

The proof of theorem 3. $G$ has a compact subgroup $K$ which is transitive on $M$ (see Montgomery [5]). Since the isotropy subgroup $K_{x}$ at a point $x \in M$ is a compact subgroup of $G$, the linear isotropy group induced from $K_{x}$ on the tangent space $T_{x}$ of $M$ at $x$ is an isometry group. Then there exists a unique metric on $M$ which on $T_{x}$ coincides with the given and with respect to which $K$ is an isometry group. Give this metric to the manifold $M$, then we get the space $M^{*}$ required.

Remark. If, moreover, $M$ is conformally flat, $M$ is conformally equivalent to the sphere (i. e. the complete simply connected space of positive constant curvature), according to a more general theorem of Kuiper [4] or to the corollary of theorem 6 in $\S 2$. 


\section{$\S 2$. The structure of the Lie algebra $C^{I}(M)$.}

Let $u$ be an infinitesimal conformal transformation on $M$. Putting

we have

$$
£_{u} g=2 \phi g,
$$

$$
\begin{aligned}
\nabla_{k} \nabla_{j} u^{i} & =\mathfrak{£}_{u}\left\{\begin{array}{l}
i \\
k j
\end{array}\right\}-R_{j k a}^{i} u^{a} \\
& =\delta_{k}^{i} \phi_{j}+\delta_{j}^{i} \phi_{k}-\phi^{i} g_{k j}-R_{j k a}^{i} u^{a}
\end{aligned}
$$

where

$$
\phi_{j}=\partial_{j} \phi
$$

and

where

$$
\nabla_{j} \phi_{i}=£_{u} L_{j i} /(n-2)
$$

$$
L_{j i}=-K_{j i}+k g_{j i} / 2(n-1) .
$$

Hence, by virtue of the theorem of the unique existence in the theory of differential equations, $u$ is uniquely determined by the values of $u^{i}, \nabla_{j} u^{i}, \phi$ and $\phi_{i}$ at a point of $M$. If $u$ and $v$ are infinitesimal conformal transformations satisfying $£_{u} g=2 \phi g$ and $£_{v} g=2 \psi g$, then $[u, v]$ belongs to $C^{I}(M)$. Putting $\mathfrak{£}_{[u, v]} g=2 \chi g$, we have

$$
\begin{aligned}
& {[u, v]^{i} }=u^{a} \nabla_{a} v^{i}-v^{a} \nabla_{a} u^{i}, \\
& \nabla_{j}[u, v]^{i}=\left(\nabla_{j} u^{a}\right) \nabla_{a} v^{i}-\left(\nabla_{j} v^{a}\right) \nabla_{a} u^{i}-u^{a}\left(£\left\{\begin{array}{c}
i \\
a j
\end{array}\right\}-R_{a j b}^{i} v^{b}\right) \\
&-v^{a}\left(£_{u}\left\{\begin{array}{c}
i \\
a j
\end{array}\right\}-R_{a j b}^{i} u^{b}\right), \\
& \chi= u^{a} \psi_{a}-v^{a} \phi_{a} \\
& \chi_{i}=\left(\nabla_{i} u^{a}\right) \psi_{a}+u^{a} £_{v} L_{i a} /(n-2)-\left(\nabla_{i} v^{a}\right) \phi_{a} \\
&-v^{a} £_{u} L_{i a} /(n-2) .
\end{aligned}
$$

These relations give the structure of Lie algebra $C^{I}(M)$, which tells us various facts. But we are especially interested in conformally flat spaces, in view of the reduction to the isometry discussed in $\S 1$. From now on we assume that $M$ is conformally flat. For a fixed point $p \in M, M$ is then conformally equivalent to a space $M^{*}$, and some neighborhood $U$ of $p$ corresponds to a neighborhood in $M^{*}$ which is isometric onto the Euclidean space. Thus we can suppose $\left\{\begin{array}{c}i \\ a j\end{array}\right\}=0$ in $U$, so long as we are concerned with the structure of $C^{I}(M)$. Hence

$$
u \in C^{I}(M) \rightarrow\left(\begin{array}{ccc}
-\phi & u^{i} & 0 \\
-\phi_{j} & \partial_{j} u^{i}-\delta_{j}^{i} & u^{j} \\
0 & -\phi_{i} & \phi
\end{array}\right) \quad \text { evaluated at } p
$$


is an isomorphism of $C^{I}(M)$ into the Lie algebra of the general linear group $G L(n+2)$ of degree $n+2$. The $n$-Möbius group $M(n)$ is by definition the subgroup of $G L(n+2)$ consisting of all the linear transformations which leave invariant the quadratic form $\sum_{i=2}^{n+1}\left(x^{i}\right)^{2}-2 x^{1} x^{n+2}$ (with respect to a base). The Lie algebra of $M(n)$ is called the $n$-Möbius algebra $M^{L}(n)$. Now it is easy to prove

Theorem 4. For any conformally flat space $M, C^{I}(M)$ (a fortiori $C^{L}(M)$ ) is isomorphic to a subalgebra of n-Möbius algebra. The correspondence is given by (2.5).

This theorem enables us to use the matrix calculus in detailed researches of the structure of $C^{I}(M)$ and so useful in the proofs of the following theorems.

Remark. A conformally flat space $M$ is covered by open subsets $\left\{U_{\lambda}\right\}$ such that $C^{I}\left(U_{\lambda}\right)$ is isomorphic onto $M^{L}(n)$, as Sasaki and later Taub found (see Yano [8]).

The next theorem might be known (see Kuiper [4]), and only the proof is new.

Theorem 5. The sphere $S^{n}$ is the unique Riemannian space which admits the Lie algebra of the conformal transformation group isomorphic to the n-Möbuis algebra:

$$
C^{I}\left(S^{n}\right)=C^{L}\left(S^{n}\right) \cong M^{L}(n) .
$$

Proof. Since $S^{n}$ is of constant curvature, $S^{n}$ is conformally flat. Hence the system (2.1), (2.2), (2.3) and (2.4) to characterize a $u \in C^{I}(M)$ is completely integrable (cf. the remark above). $S^{n}$ being simply connected, these equations are globally solvable, i. e. $C^{I}\left(S^{n}\right)$ is isomorphic to $M^{L}(n)$. The compactness of $S^{n}$ implies $C^{I}\left(S^{n}\right)=C^{L}\left(S^{n}\right)$. The uniqueness will be proved later.

Theorem 6. A conformally flat space $M$ which admits a transitive connected conformal transformation group $G$ has the same (but for the conformal equivalence) universal covering space as an open submanifold of the sphere.

Proof. We can assume $M$ simply connected. For a fixed point $p \in M$, there is a neighborhood $U$ of $p$ and a conformal diffeomorphism $\omega_{U}$ of $U$ onto an open subset of $S^{n}$, for $M$ is conformally flat. $\omega_{U}$ induces an isomorphism $d \Omega$ of the Lie algebra $G^{L}$ of $G$ into $C^{L}\left(S^{n}\right)$. Note that the isotropy subalgebra of $G^{L}$ corresponds to that of $C^{L}\left(S^{n}\right)$ under $d \Omega$. The universal covering group $\tilde{G}$ of $G$ operates naturally on $M$. The kernel $\pi_{1}(G)$ of the projection $\tilde{G} \rightarrow G$ consists of the identity transformation. $d \Omega$ defines a homomorphism $\widetilde{\Omega}$ of $\tilde{G}$ into $C\left(S^{n}\right)$. The isotropy subgroup $\tilde{G}_{p}$ of $\tilde{G}$ at $p$ is connected, for $\tilde{G}$ and $M$ are simply connected. Thus $\tilde{G}_{p}$ is mapped to the isotropy subgroup $C_{q}\left(S^{n}\right)$ at $q=\omega_{U}(p)$. Since $\pi_{1}(G) \subset \tilde{G}_{p}$ is a normal subgroup 
of $G$ and $C\left(S^{n}\right)$ is effective, $\tilde{\Omega}\left(\pi_{1}(G)\right)$ is the neutral element of $C\left(S^{n}\right)$. Therefore $\tilde{\Omega}$ induces a homomorphism $\Omega$ of $G$ into $C\left(S^{n}\right)$, which is a local isomorphism just as well as $\tilde{\Omega}$. The isotropy group $G_{p}$ of $G$ at $p$ is mapped by $\Omega$ into $C_{q}\left(S^{n}\right)$. Thus $\Omega$ induces a local diffeomorphism $\omega$ of $M=G / G_{p}$ onto $\Omega(G) / \Omega\left(G_{p}\right) \subset S^{n}$. $\Omega(G) / \Omega\left(G_{p}\right)$ is an open subspace of $S^{n}$, for among the elements near the neutral element of $G$ only those of $G_{p}$ are mapped into $C_{p}\left(S^{n}\right)$ by $\Omega$. The restriction $\omega \mid U$ of $\omega$ to $U$ coincides with $\omega_{U}$. Hence $\omega$ is locally conformal, in particular locally diffeomorphic.

Remark. In this theorem "an open submanifold of $S^{n}$ " cannot be replaced by $S^{n}$ itself, because a space with a transitive conformal group is not always complete with respect to the so-called normal conformal connection (nor the Levi-Civita connection) (e.g. the Euclidean space).

Remark. N.H. Kuiper proved that a conformally flat space can "conformally be developped" into $S^{n}$ in a unique way ([4]). Our theorem 6 sharpens his result in the case of transitive conformal group.

Corollary. A compact simply connected space $M$ which admits a connected transitive conformal transformation group is conformally equivalent to the sphere, if $M$ is conformally flat. This was announced at the end of $\S 1$.

Proof. By virtue of theorem 6, the compact space is a covering of an open submanifold $V$ of $S^{n}$. Hence $V$ is compact and closed in $S^{n}$. Thus $V=S^{n}$. $S^{n}$ being simply connected, $M$ must coincide with $S^{n}$.

The proof of the uniqueness in theorem 5 .

Let $M$ be a Riemannian space with $C^{L}(M)$ isomorphic onto $M^{L}(n)$. Denote by $G$ the neutral component of $C(M)$. Since the Lie algebra $G^{L} \subset$ $C^{L}(M)$ is transitive, $G$ is transitive. On account of theorem 6 , the universal covering $\tilde{M}$ of $M$ is conformally equivalent to that of an open submanifold $V$ of $S^{n}$. Observing the proof of that theorem, we see that $G$ is locally isomorphic onto $C(V)$, hence $C(V)$ has the same dimension as $M(n)$ locally isomorphic to $C\left(S^{n}\right)$. From this follows easily $V=S^{n}$, and so $M$ is conformally equivalent to $S^{n}$. We identify these two spaces. The 1-dim. homotopy group $\pi_{1}(M)$ of $M$ is a group of isometries of $\tilde{M}=S^{n}$, which is contained in the centralizer of the neutral component of $C\left(S^{n}\right)$. (Note that, because of $\operatorname{dim} C^{L}(\tilde{M})=\operatorname{dim} C^{L}(M), C^{L}(\tilde{M})$ is induced from $C^{L}(M)$ by the projection: $\tilde{M} \rightarrow M$ and $C^{L}(\tilde{M})$ is thus invariant by $\left.\pi_{1}(M)\right)$. Now in order to complete the proof of theorem 5 , it is sufficient to show that the center of $C\left(S^{n}\right)$ reduces to the identity. But this is a direct consequence of the classical fact in Möbius geometry that for any point $p \in S^{n}$ there is a transformation $\tau$ in the neutral component of $C\left(S^{n}\right)$ which fixes just one point $p$. (Take as $\tau$ the so-called elation with center $p$ ). $\left(\pi_{1}(M)\right.$ must commute with each such $\tau$ ). 
The isometry group does not contain such a transformation, and the following theorem holds (see also Obata [7]).

TheоReм 7. An n-dimensional Riemannian space $M$ which admits the isometry group of dimension $n(n+1) / 2$ is a complete simply connected space of constant curvature or the projective space, and vice versa, where the projective $P^{n}$ means the Riemannian space obtained from the sphere $S^{n}$ naturally imbedded in $(n+1)$-dimensional Euclidean space by identifying every two points at the ends of diameters, the metric being induced in such a way that the projection: $S^{n} \rightarrow$ $P^{n}$ is a local isometry.

Proof. It is known that the universal covering space $\tilde{M}$ is a complete simply connected space of constant curvature. $\pi_{1}(M)$ is a transformation group $\subset I(\tilde{M})$ of $\tilde{M}$ contained in the centralizer of the neutral component of $I(M)$, which holds in an analogous way as in the proof given above of the uniqueness.

Case I: $M$ with positive curvature. Then we consider $M$ as the sphere in the $(n+1)$-dimensional Euclidean space $E^{n+1}$ with center at the origin. The neutral component of $I(\tilde{M})$ is the special orthogonal group $S O(n+1)$, which consists of rotations around the origin of $E^{n+1}$. A transformation in $\pi_{1}(M)$ is an isometry of the sphere, and hence this is extended to $E^{n+1}$, i. e. it belongs to the orthogonal group $O(n)$. Now the centralizer of $S O(n)$ in $O(n)$ consists of the identity and the symmetry with respect to the origin. When $\tilde{M} \neq M, M$ must therefore be the real projective space.

Case II : $M$ with non-positive curvature. Any two points of $M$ are joined with just one geodesic. The proof is easier when $M$ has even dimensions. Then for any fixed point $p \in M$ there exists a rotation $\rho$ around $p$ and which belongs to the neutral component $I_{c}(M)$ of $I(M)$. Hence $\rho$ does not fix any point of $M$ except $p$. Therefore $\pi_{1}(M)$ reduces to the identity. When $M$ has odd dimensions, take two rotations $\rho$ and $\sigma$ in $I_{c}(M)$ such that there is not any geodesic starting from $p$ which is invariant under $\rho$ and $\sigma$. There is not any point except $p$ in $M$ which is fixed by $\rho$ and $\sigma$. Hence $\pi_{1}(M)$ $=\{e\}$, that is $M=\tilde{M}$. Conversely such $M$ 's and $P^{n}$ admit the isometry group of dimension $n(n+1) / 2$, as is easily verified.

REMARK. In the same way a conformal transformation which commutes with the neutral component of $I(M)$ is an identity or, if $M$ is of positive curvature, a "symmetry" in the above sense. This fact will be used later.

\section{§ 3. Transitive conformal groups of higher dimensions.}

The isotropy algebra $C_{p}^{L}(M)$ at $p \in M$ of the conformal transformation group $C(M)$ is naturally represented on the tangent space $T_{p}$ of $M$ at $p$, 
the representation denoted by $d$ is not faithful in general. But we have

Proposition 8. Let $C(M)$ be a transitive conformal transformation group of a conformally flat space $M$. If the Lie algebra $C^{L}(M)$ is not isomorphic to the n-Möbius algebra, the isotropy subalgebra $C_{p}^{L}(M)$ of $C(M)$ is faithfully represented on the tangent space naturally.

Proof. Let $K^{L}$ be the kernel of the representation $d$ on the tangent space. Each element $u$ of $K^{L}$ satisfies

$$
u^{i}(p)=0, \quad \nabla_{j} u^{i}(p)=0, \quad \phi(p)=0
$$

where $\phi$ is given by $£_{u} g=2 \phi g$ on $M$. Suppose that $K^{L}$ is not trivial and $u \neq 0$. We can assume $\partial_{j} \phi(p)=\delta_{j 1} . C(M)$ being transitive, there exist $v_{(1)}$, $\cdots, v_{(n)} \in C^{L}(M)$ such that we have $v_{(k)}{ }^{i}(p)=\delta_{k}^{i}$. From the study of the structure of $C^{L}(M)$ in $\S 1$, we deduce that

1) $\left[u, v_{(k)}\right]$ belongs to the isotropy subalgebra $C_{p}^{L}(M)$ and the elements $\left[u, v_{(k)}\right](k=2, \cdots, n)$ generate (or, more in detail, $\left[u, v_{(k)}\right]$ and $\left[\left[u, v_{(k)}\right],\left[u, v_{(l)}\right]\right]$ $(k, l=2, \cdots, n)$ span) a subalgebra whose $d$-image is the Lie algebra $O^{L}(n)$ of the orthogonal group.

2) $u$ and $\left[\left[u, v_{(k)}\right], u\right](k=2, \cdots, n)$ belong to $K^{L}$ and are linearly independent and finally $v_{(k)}(k=1, \cdots, n),\left[u, v_{(1)}\right]$ and those elements in 1) and 2) span an $(n+1)(n+2) / 2$ dimensional linear subspace of $C^{L}(M)$. But this dimension is equal to that of $M^{L}(n)$. Thus $C^{L}(M)$ is isomorphic to $M^{L}(n)$, by virtue of theorem 4 .

TheOREM 9. If a space $M$ with dimension $n>4$ admits a subgroup $G$ of $C(M)$ which is transitive and dimension greater than $n(n-1) / 2+2$, then one of the following 5 cases occurs (conformal equivalence being neglected):

1) $M$ is the sphere $S^{n}$ and $G \cong M(n)$, hence $\operatorname{dim} G=(n+1)(n+2) / 2$,

2) $M$ is the Euclidean space $E^{n}$ and $G \cong C(M) \cong$ the homothetic transformation group, hence $\operatorname{dim} G=n(n+1) / 2+1$,

3) $M$ is $S^{n}$ or the real projective space and $G \cong I\left(S^{n}\right)$, hence $\operatorname{dim} G=n(n+1) / 2$,

4) $M$ is $E^{n}$ and $G \cong I\left(E^{n}\right)$, hence $\operatorname{dim} G=n(n+1) / 2$,

5) $M$ is the hyperbolic space $H^{n}$ and $G \cong I\left(H^{n}\right)$, hence $\operatorname{dim} G=n(n+1) / 2$. In the above, $\cong$ means local isomorphism.

REMARK. $H^{n}$ is obtained, if in the inner part of the hyper-sphere with center at the origin and radius $r$ in $E^{n}$ we define the metric by $r^{2} \Sigma\left(d x^{i}\right)^{2} /$ $\left[r^{2}-\Sigma\left(x^{j}\right)^{2}\right]$. Apparently $H^{n}$ is conformally equivalent to the interior of the hypersphere, but not onto $E^{n}$, because we have $C\left(H^{n}\right) \cong C\left(E^{n}\right)$ by virtue of theorem 9 above.

The proof of theorem 9 will be completed after several lemmas. First $M$ is conformally flat, as was remarked before.

Lемма 3.1. Under the hypothesis in theorem 9, the isotropy subgroup is locally isomorphic onto the orthogonal group $O(n)$ or the direct product of $O(n)$ 
and a 1-parameter group.

Proof. The isotropy subalgebra $C_{p}^{L}(M)$ contains $C_{*}^{L}=\left\{u \in C^{L}(M): u(p)\right.$ $=0, \phi(p)=0\}$ and has dimension $\leqq \operatorname{dim} C_{*}^{L}+1$. Thus, by proposition 8 , we have $\operatorname{dim} C_{*}^{L} \geqq n(n-1) / 2-(n-1)$. $C_{*}^{L}$ can be regarded as a subalgebra of the Lie algebra $O^{L}(n)$ of $O(n) . \quad C_{*}^{L}$ generates a connected subgroup $C_{*} \subset O(n)$. According to Montgomery and Samelson [7], $C_{*}$ is dense in $O(n)$. Further the inner automorphism of $O(n)$ corresponding to the elements of $C_{*}$ leave $C_{*}^{L}$ invariant, if they are induced on $O^{L}(n)$. Thus the special orthogonal group $S O(n)$ leaves $C_{*}^{L}$ invariant. Since $S O(n)$ is simple, $C_{*}^{L}=O^{L}(n)$. When $C_{p}^{L}(M) \neq C_{*}^{L}, C_{p}^{L}(M)$ is the direct sum of $C_{*}^{L}$ and a 1 -dimensional subalgebra.

We fix a point $p \in M$ and a coordinate system valid in a neighborhood $U$ of $p$ such that $C^{I}(U)\left(\supset C^{L}(M)\right)$ is isomorphic onto $M^{L}(n)$. We can suppose $U \subset M$ is flat and the fixed coordinate system is Cartesian. We choose a base of $M^{L}(n)\left\{e^{i}, f^{i}, h, E_{i}{ }^{j}(i, j=1, \cdots, n) i<j\right\}$ such that

$$
\iota: u \in C^{\mathbb{I}}(U) \rightarrow u^{k}(p) e_{k}-\partial_{k} \phi(p) f^{k}+\phi(p) h-\left(\partial_{j} u^{i}(p)-\phi(p) \delta_{j}^{i}\right) E_{i}^{j}
$$

is the isomorphism (2.5) of $C^{I}(U)$ onto $M^{L}(n)$, where $\phi$ satisfies $£_{u} g=2 \phi g$. We shall neglect $\iota$ (i. e. we shall consider $C^{L}(M) \subset C^{I}(U)=M^{L}(n)$ ) in the sequel. We assume that $G$ is not locally isomorphic to $M(n)$.

Lemma 3.2. There exists a real number a such that all $a\left(f^{i}-f^{j}\right)+E_{i}^{j}(i, j$ $=1, \cdots, n)$ belong to $C^{L}(M)$.

Proof. We shall give the proof in two ways. By the second method we shall quickly prove lemmas 2 and 3 at the same time, with the use of a theorem on the maximal compact subgroup. It will be found after the proof of lemma 3. Now we begin with the first proof, in which we shall not adopt Einstein's summation convention. The commutator subalgebra of $C_{p}^{L}(M)$ coincides with $C_{*}^{L}$ in the proof of lemma 3.1, hence with $O^{L}(n)$. Thus $C_{*}^{L}$ has the base of the form $\sum_{k=1}^{n} \phi_{k}(i j) f^{k}+E_{i}{ }^{j}$. For any fixed $i_{1}$ and $j_{1}\left(i_{1}<j_{1}\right)$, take any $i_{2}$ and $j_{2}$ such that $\left(i_{1}-i_{2}\right)\left(i_{1}-j_{2}\right)\left(j_{1}-i_{2}\right)\left(j_{1}-j_{2}\right) \neq 0$ and $i_{2}<j_{2}$.

Then we have

$$
\begin{aligned}
& {\left[\sum_{k=1}^{n} \phi_{k}\left(i_{1} j_{1}\right) f^{k}+E_{i_{1}}^{j_{1}}, \sum_{k} \phi_{k}\left(i_{2} j_{2}\right) f^{k}+E_{i_{2}}^{j_{2}}\right]} \\
& \quad=\phi_{j_{1}}\left(i_{2} j_{2}\right) f^{i_{1}}-\phi_{i_{1}}\left(i_{2} j_{2}\right) f^{j_{1}}-\phi_{j_{1}}\left(i_{1} j_{1}\right) f^{i_{2}}+\phi_{j_{1}}\left(i_{1} j_{1}\right) f^{j_{2}}
\end{aligned}
$$

from the relation

$$
\left[E_{i}{ }^{j}, f^{k}\right]=\delta_{k}{ }^{j} f^{i}-\delta_{i}{ }^{k} f^{j} \text { and }\left[f^{i}, f^{k}\right]=0 .
$$

The right hand side must vanish by virtue of proposition 8 . Thus we have

$$
\phi_{k}(i j)=0 \quad \text { for } k \neq i, j
$$

or 


$$
\sum_{k} \phi_{k}(i j) f^{k}+E_{i}^{j}=\phi_{i}(i j) f^{i}+\phi_{j}(i j) f^{j}+E_{i}^{j} .
$$

From (3.1) and (3.2) we obtain, for $i<j_{1}<j_{2}$,

$$
\begin{array}{r}
{\left[\sum_{k} \phi_{k}\left(i j_{1}\right) f^{k}+E_{i}^{j_{1}}, \sum_{k} \phi_{k}\left(i j_{2}\right) f^{k}+E_{i}^{j_{2}}\right]} \\
=-E_{j_{1}}^{j_{2}}+\phi_{i}\left(i j_{1}\right) f^{j_{3}}-\phi_{i}\left(i j_{2}\right) f^{j_{1}} .
\end{array}
$$

By virtue of proposition 8 and (3.3), this right hand side is equal to

and we have

$$
-E_{j_{1}}^{j_{2}}-\phi_{j_{1}}\left(j_{1} j_{2}\right) f^{j_{1}}-\phi_{j_{2}}\left(j_{1} j_{2}\right) f^{j_{2}}
$$

$$
\phi_{i}\left(i j_{2}\right)=\phi_{j_{1}}\left(j_{1} j_{2}\right) \text { and } \phi_{i}\left(i j_{1}\right)=-\phi_{j_{2}}\left(j_{1} j_{2}\right)
$$

Further from the relation

follows

$$
\begin{aligned}
& {\left[-E_{j_{1}}^{j_{2}}-\sum_{k} \phi_{k}\left(j_{1} j_{2}\right) f^{k}, E_{i}^{j_{1}}+\sum_{k} \phi_{k}\left(i j_{1}\right) f^{k}\right]} \\
& =E_{i}^{j_{2}}+\phi_{j_{1}}\left(i j_{1}\right) f^{j_{2}}+\phi_{j_{1}}\left(i j_{2}\right) f^{i}
\end{aligned}
$$

$$
\phi_{j_{2}}\left(i j_{2}\right)=\phi_{j_{1}}\left(i j_{1}\right) \text {. }
$$

(3.4) and (3.5) enable us to conclude that $\phi_{i}\left(i j_{1}\right)=-\phi_{j_{1}}\left(i j_{1}\right)$ is independent of $\left(i, j_{1}\right)$ such that $i<j_{1}$.

Lemma 3.3. We can assume that the number $a$ in the preceding lemma is zero.

Proof. The inner automorphism of $M(n)$ given by $\exp \left(-a \sum_{i=1}^{n} f^{i}\right)$ induces an automorphism of $M^{L}(n)$ by which $a\left(f^{i}-f^{j}\right)+E_{i}{ }^{j}$ is mapped to $E_{i}{ }^{j}$. (We can consider that this automorphism of $M(n)$ corresponds to a change of coordinates and a conformal change of the metric. But these changes do not matter for our purpose.)

Now we give the second proof of Lemmas 2 and 3. Let $M^{L}$ be the subalgebra $\left\{\phi_{k} f^{k}+U_{j}{ }^{i} E_{i}{ }^{j}\right\}$ is $M^{L}(n) . M_{*}^{L}$ is isomorphic onto the Lie algebra $I^{L}\left(E^{n}\right)$ of the isometry group of the Euclidean space. $C_{*}^{L} \subset M_{*}^{L}$ is isomorphic to the subalgebra $R^{L}=\left\{U_{j}{ }^{i} E_{i}^{j}\right\}$, which is isomorphic to the rotation algebra of $I^{L}\left(E^{n}\right)$.

Hence $C_{*}^{L}$ and $R^{L}$ generate the maximal compact subgroups of the connected group $M_{*}$ generated by $M_{*}^{L}$. Therefore $C^{L}$ is transformed onto $R^{L}$ by an inner automorphism of $M_{*}$, according to Malcev-Iwasawa (see [3]). This automorphism can be extended to $M(n)$ and leaves the isotropy subalgebra invariant.

Remark. In the above arguments we have found that in the isometry group of the $n$-dimensional Euclidean space there are $n(n-1) / 2$-dimensional subgroups different from the rotation group and that all such groups which 
do not contain any parallel displacement are compact.

Lemma 3.4. We can assume that $C^{L}(M)$ is spanned by the basis of the isotropy subalgebra $C^{L}(M)$ and the elements of the form $p f^{i}+\psi(i) h+e_{i}$ where $p= \pm 1$ or 0 (independent of $i$ ).

Proof. $C^{L}(M)$ contains the elements of the form $c_{k}(i) f^{k}+\psi(i) h+e_{i}$ and $E_{i}{ }^{j}$

$$
\begin{aligned}
{\left[E_{i}{ }^{j}, c_{h}(k) f^{h}\right.} & \left.+\psi(k) h+e_{k}\right] \\
& =c_{j}(k) f^{i}-c_{i}(k) f^{j}+\delta_{k j} e_{i}-\delta_{k i} e_{j} .
\end{aligned}
$$

From this and proposition 8 , we conclude that there is a number $c$ such that $c_{i}(k)=\delta_{i k} c$. When $c \neq 0$, consider an inner automorphism of $M^{L}(n)$ defined by $\exp (-\log \sqrt{|c|} h)$. The images of $c f^{i}+\psi(i) h+e_{i}$ and $E_{i}{ }^{j}$ are $\sqrt{|c|}\left( \pm f^{i}+e_{i}\right)+\psi(i) h$ and $E_{i}^{j}$ respectively. Replace $\sqrt{|c|}\left( \pm f^{i}+e_{i}\right)+\psi(i) h$ by $\pm f^{i}+e_{i}+\psi(i) / \sqrt{|c|} h$.

The proof of theorem 9 .

Case 1: $C_{p}^{L}(M) \neq O^{L}(n) . \quad C_{P}^{L}(M)$ is spanned by the elements of the form $\phi_{i} f^{i}+h$ and $E_{i}{ }^{j}$. By the inner automorphism considered in the proof of lemma 3.4, the coefficients $\psi(k)$ were not changed. Hence in the present case we can suppose $\psi(k)=\delta_{k}^{1} \psi(1), \psi(1) \neq 0$. From $\left[E_{1}^{2}, p f^{2}+e_{2}\right]=p f^{1}+e_{1}$, we have $h$ in $C_{p}^{L}(M)$. Thus $C^{L}(M)$ is spanned by $E_{i}^{j}, h$ and $p f^{k}+e_{k}$. From $\left[h, p f^{k}+e_{k}\right]=p f^{k}-e_{k}$, we deduce that $p=0$, because otherwise $f^{k} \in C^{L}(M)$, contrary to proposition 8 and the assumption. Thus $C^{L}(M)$ is isomorphic to $C^{L}\left(E^{n}\right)$ and the universal covering $\tilde{M}$ of $M$ is conformally equivalent to the Euclidean space $E^{n}$. Identifying $\tilde{M}$ with $E^{n}$, we can consider $\pi_{1}(M)$ as a conformal transformation group which is contained in the centralizer of the neutral component of $C\left(E^{n}\right)$. Refering to the remark at the end of $\S 2$, we conclude that $M$ is conformally equivalent to $E^{n}$.

Case 2: $\quad C_{p}^{L}(M)=O^{L}(n)$. From $\left[E_{i}{ }^{j}, p f^{j}+\psi(j) h+e_{j}\right]=p f^{i}+e_{i}$ and $\left[E_{i}{ }^{j}, p f^{i}\right.$ $\left.+\psi(i) h+e_{i}\right]=-\left(p f^{j}+e_{j}\right)$, we have $E_{i}{ }^{j}$ and $p f^{k}+e_{k}$ as the basis of $C^{L}(M)$. Then $C^{L}(M)$ is isomorphic to the Lie algebra of the isometry group of the elliptic, Euclidean and hyperbolic geometry according as $p$ is $-1,0$ and +1 respectively. Hence we can prove in the same way as above that $M$ is conformally equivalent to the Euclidean, hyperbolic, spherical or projective spaces. Finally we prove that these spaces are not conformally equivalent to each other. Only the spaces which are homeomorphic are the Euclidean space $E^{n}$ and the hyperbolic space $H^{n}$. We can prove that $C\left(E^{n}\right)$ is not isomorphic to $C\left(H^{n}\right)$ by using the known structure of the isometry groups of these symmetric spaces.

But here we give another proof. According to Yano and Nagano [11], for an Einstein space $M$ which is neither the sphere nor the Euclidean 
space, we have $C^{L}(M)=I^{L}(M)$. Thus $C^{L}\left(H^{n}\right)=I^{L}\left(H^{n}\right)$. On the other hand, evidently $C^{L}\left(E^{n}\right) \neq I^{L}\left(E^{n}\right) \quad\left(E^{n}\right.$ admits a $l$-parameter group of homothetic transformations). From $\operatorname{dim} I^{L}\left(H^{n}\right)=\operatorname{dim} I^{L}\left(E^{n}\right)=n(n+1) / 2$, we have $C\left(E^{n}\right)$ $\neq C\left(H^{n}\right)$. Therefore $E^{n}$ is not conformally equivalent to $H^{n}$.

\section{$\S 4$. Intransitive conformal groups of higher dimensions.}

In this section we shall prove the following theorem 10, which, combined with theorem 9 , gives the complete list of the spaces which admit conformal groups with dimension $>n(n-1) / 2+2$ and the structure of these groups for $n>5$.

THEOREM 10. If a connected Riemannian space $M$ with dimension $n>5$ admits an intransitive conformal transformation group $G \subset C(M)$ with $\operatorname{dim} G>$ $n(n-1) / 2+2$, then 1) $M$ is conformally equivalent to the sphere and 2) two cases are possible: (1) G leaves invariant just one point and the complementary open subspace which is conformally equivalent to the Euclidean space $E^{n}$ and $G$ is locally isomorphic to $C\left(E^{n}\right)$ or $I\left(E^{n}\right)$ and (2) G leaves invariant just one hypersphere operates effectively on the hypersphere and the two open submanifolds conformally equivalent to the hyperbolic space $H^{n}$ with $C\left(H^{n}\right)$ locally isomorphic to $G$.

The rest of this section will be devoted to the proof of this theorem.

Lemma 4.1. If we have $G \subset C(M)$ and $\operatorname{dim} G>n(n-1) / 2+1$, each orbit has dimensions $\geqq n-1$ or $=0$.

Proof. $M$ is then conformally flat. If an orbit is of dimension $k$, for a point $p$ of this orbit $(=G(p)=$ the image of $p$ by $G)$, the subspace $\{u(p)$; $\left.u \in C^{L}(M)\right\}$ of the tangent space at $p$ is of dimension $k$ and invariant by the isotropy subalgebra. Hence the observation of the structure of $C^{L}(M)$ surveyed in $\S 3$ shows us $n(n-1) / 2+1 \leqq \operatorname{dim} C^{L}(M) \leqq k+k(k-1) / 2+(n-k)$ $\times(n-k-1) / 2+n+1$, from which we have $k \geqq n-1$ or $=0$.

LeMMA 4.2. If an $r$-dimensional conformal group $G \subset C(M)$ of a conformally flat space $M$ leaves invariant an $(n-1)$-dimensional subspace $N^{n-1} \subset M$, then the effective group induced on $N^{n-1}$ is of dimension $\geqq r-1$.

Proof. Putting $G_{N}^{L}=\left\{u \in C(M) ; u=0\right.$ on $\left.N^{n-1}\right\}$. We have to show $\operatorname{dim}$ $G^{L} \leqq 1$. Take an arbitrary point $p$ on $N^{n-1}$ and a normal coordinate system $\left(x^{i}\right)$ valid in a neighborhood which is assumed to be flat without loss of generality and such that $N^{n-1}$ satisfies $d x^{1}=0$ at $p$. If $u \in G_{N}^{L}$, we have not only $u^{i}(p)=0$ but $\partial_{\alpha} u^{i}=0(1 \leqq i \leqq n, 2 \leqq \alpha \leqq n)$, in particular $\partial_{2} u^{2}(p)=0$. From $£_{u} g=2 \phi g$ which means $\partial_{j} u^{i}+\partial_{i} u^{j}=2 \phi \delta_{j}{ }^{i}$, we conclude $\phi(p)=0$, hence $\partial_{1} u^{1}(p)$ $=0$ and $\partial_{i} u^{\alpha}=-\partial_{\alpha} u^{i}=0$ at $p$. Thus all the components $\partial_{j} u^{i}$ vanish at $p$. Since $\phi=0$ holds on $N^{n-1}$, we have $\partial_{\alpha} \phi(p)=0(2 \leqq \alpha \leqq n)$. Therefore we have $\operatorname{dim} G_{N}^{L} \leqq 1$ (see theorem 4). 
Proposition 11. Assume that a family $\Phi$ of hypersurfaces is defined in a neighborhood $U$ of a point $p$ in a space $M$ by a gradient vector field which does not vanish in $U$.

1) If a conformal transformation $\tau$ of $M$ leaves invariant the point $p$ and each hypersurface $\in \Phi$, then the differential $d \tau$ is an isometry of the tangent space at $p$.

2) Analogously, if an infinitesimal conformal transformation $u\left(£_{u} g=2 \phi g\right)$ leaves invariant $p$ and each hypersurface in $\Phi$, then we have $u(p)=0$.

Proof. 1) The trajectory $\gamma$ of the normal vectors of $\Phi$ which passes through $p$ is invariant by $\tau$, for $\tau$ preserves the orthogonality and fixes $p$. $\gamma$ has just one point common with each hypersurface $\in \Phi$ near $p$. Hence $r$ is left invariant point-wise by $\tau$, which implies that the normal vector of the hypersurface at $p$ is invariant by $\tau$. Therefore $\tau$ induced on the tangent space is an isometry.

2) Denote by $\psi$ the function defined near $p$ in terms of which $\Phi$ is given by $\psi=$ const. From $£_{u} \nabla_{i} \psi=\nabla_{i} £_{u} \psi=0$, we obtain $\left(\nabla_{a} \psi\right) \nabla_{i} u^{a}=0$ at $p$. Hence we get at $p$

$$
\phi\left(\nabla^{a} \psi\right)\left(\nabla_{a} \psi r\right)=\left(\nabla^{a} \psi\right)\left(\nabla_{b} u_{a}\right) \nabla^{b} \psi=0 .
$$

$\nabla_{a} \psi(p)$ being non-zero, we conclude that $\phi(p)=0$. Now we prove theorem 10. By the assumption of intransitivity and lemma 4.1 we have only to investigate following two cases:

(A) All the orbits are of dimension $\geqq n-1$, and there exists an $(n-1)$ dimensional orbit.

(B) There exists a zero-dimensional orbit.

Case (A). There exists an $n$-dimensional orbit $O^{n}$, because otherwise, due to lemma 4.2 and proposition 11 , the orbits are conformally equivalent to $(n-1)$ dimensional spaces with an isometry group of dimensions $>n(n-1) / 2$, which is never possible. The $n$-dimensional orbit $O^{n}$ is an open subspace of $M$, and hence $O^{n}$ is not compact. By virtue of theorem 9, each connected component $O_{0}^{n}$ of $O^{n}$ is conformally equivalent to the Euclidean or hyperbolic space, and $\operatorname{dim} G=n(n+1) / 2$ or $n(n+1) / 2+1$. Its boundary is an $(n-1)-$ dimensional orbit $O^{n-1}$ on which $G$ induces an $n(n+1) / 2$-dimensional conformal group, by virtue of theorem 9 and lemma 4.2. Thus $O^{n-1}$ is comformally equivalent to the sphere, and $G$ induced on $O^{n-1}$ is locally isomorphic to $M(n-1)$. If $O_{0}^{n}$ is comformally equivalent to the Euclidean space, then $I^{L}\left(E^{n}\right)$ must be isomorphic to $M^{L}(n-1)$. But this is impossible, for the former has an $n$-dimensional ideal (corresponding to the parallel displacements) and the latter not (in fact the latter is simple as the Lie algebra of a Lorentz group). Consider a coordinate system as in the proof of lemma 4.2 near a point $p \in O^{n-1}\left(N^{n-1}\right.$ replaced by $\left.O^{n-1}\right)$. Since $G^{L}$ induced on $O^{n-1}$ 
is isomorphic to $M^{L}(n-1)$, there exists a $u \in G^{L}$ such that $\partial_{j} u^{1}(p)=0$ and $\partial_{j} \phi(p)=\delta_{j_{2}}(j=1, \cdots, n)$. Then we have $\partial_{2} \partial_{1} u^{1}(p)=\partial_{2} \phi(p) \neq 0$ and $\partial_{1} \partial_{1} u^{1}(p)$ $=\partial_{2} \partial_{2} u^{1}(p)=0$ from (2.2). Hence $u^{1} \neq 0$ at every point (near $p$ ) on some curve with a tangent vector $(1,1,0, \cdots, 0)$ at $p$. Considering the freedom in the choice of our coordinates, we conclude that $G^{L}$ is transitive on a neighborhood of $U$ of $p$ minus $U \cap O^{n-1}$. Hence there is an $n$-dimensional orbit $' O^{n}$ such that $O^{n} \cup O^{n-1} \cup{ }^{\prime} O^{n}$ forms a neighborhood of $O^{n-1}$. Repeating the above arguments, we find that also each connected component ' $O^{n}$ is conformally equivalent to the hyperbolic space $H^{n}$ and, in particular, homeomorphic to the Euclidean space $E^{n}$. Hence $O^{n} \cup O^{n-1} \cup^{\prime} O^{n}$ is homeomorphic to the sphere and is an open submanifold which is invariant by $G$. Therefore we have $M=O^{n} \cup O^{n-1} \cup{ }^{\prime} O^{n}$. (When $O^{n}$ is not connected, we have $O^{n}={ }^{\prime} O^{n}$ ). Case (B). Let $p$ be a point in $M$ invariant by $G$. Theorem 4 and the assumption on the dimension of $G$ enable us to conclude that $G \cong I\left(E^{n}\right)$ or $C\left(E^{n}\right)$ (locally). (Note that an isotropy subalgebra $C_{0}^{L}\left(S^{n}\right)$ is isomorphic to $C^{L}\left(E^{n}\right)$ ). A neighborhood of $p$ minus $p$ is contained in an $n$-dimensional orbit $O^{n}$ which is conformally equivalent to the Euclidean space, and so $O^{n} \cup\{p\}$ is homeomorphic to the sphere which implies $O^{n} \cup\{p\}=M$.

\section{University of Tokyo.}

\section{Bibliography}

[1] H. Hiramatu, On Riemannian spaces admitting groups of conformal transformations, J. Math. Soc. Japan, 9 (1957), pp. 114-130.

[2] V. Hlavatý, Zur Konformgeometrie, Proc. Acad. Amsterdam, 38 (1935), pp. 281286.

[3] K. Iwasawa, On some types of topological groups, Ann. of Math., (2) 50 (1949), pp. 507-557.

[4] N.H. Kuiper, On conformally-flat spaces in the large. Ann. of Math., 50 (1949), pp. 916-924.

[5] D. Montgomery, Simply connected homogeneous spaces, Proc. Amer. Math. Soc., 1 (1950), pp. 467-469.

[6] D. Montgomery and H. Samelson, Transformation groups of spheres, Ann. of Math., 44 (1943), pp. 454-470.

[7] M. Obata, On $n$-dimensional of homogeneous spaces of Lie groups of dimension greater than $n(n-1) / 2$. J. Math. Soc. Japan, 7 (1955), pp. 371-388.

[8] K. Yano, The theory of Lie derivatives and its application, Amsterdam, (1957).

[9] K. Yano, On $n$-dimension Riemannian spaces admitting a group of motions of order $n(n-1) / 2+1$, Trans. Amer. Math. Soc., 74 (1953), pp. 260-279.

[10] K. Yano and T. Nagano, Some theorems on projective and conformal transformations, Proc. Acad. Amsterdam, 60 (1957), pp. 451-458.

[11] K. Yano and T. Nagano, Einstein spaces admitting a one-parameter group of conformal transformations, Ann. of Math., to appear. 